\title{
Acute mesenteric venous thrombosis in a pregnant woman at 35 weeks of gestation: a case report and review of the literature
}

\author{
Xiuting Guan, Lina Huang and Liping Li (D)
}

\begin{abstract}
Background: Mesenteric venous thrombosis (MVT) is an infrequent thrombotic event that can cause devastating intestinal hemorrhagic ischemia. The mortality rate among patients with acute MVT ranges from 20 to 50\%. Occurrence of MVT in pregnancy is quite rare. In this case report, we describe a pregnant woman who presented with acute MVT at 35 weeks of gestation.

Case presentation: Our case was a 26-year-old primigravid woman at 35 weeks gestation. She presented to Guangzhou First People's Hospital with complaints of abdominal pain, nausea and vomiting. The second day after admission, she complained of more intense abdominal pain, anorexia, vomiting and abdominal distention that were out of proportion to physical signs. An emergency exploratory laparotomy was performed. The entire ileum, part of the jejunum and part of the ascending colon were gangrenous, and thromboembolism was discovered in the corresponding mesenteric veins. The necrotic intestine was resected and an end-to-end jejunum-colon anastomosis was performed. A cesarean section was performed to remove the placenta and fetus, which had expired. Histopathological analysis revealed extensive edema, hemorrhage, inflammatory infiltration and necrosis in the resected bowel, and widespread thrombosis in mesenteric venous lumens.
\end{abstract}

Conclusion: The diagnosis of MVT during pregnancy is very difficult due to its low incidence, and non-characteristic symptoms, signs and laboratory results. MVT may be the underlying cause of severe abdominal pain during pregnancy and should be included in the differential diagnosis of pregnant patients with an acute abdomen.

Keywords: Mesenteric venous thrombosis, Pregnancy, Acute abdomen, Intestinal ischemia

\section{Background}

Mesenteric venous thrombosis (MVT) is an infrequent thrombosis with an estimated incidence of only 2.7 per 100,000 person-years [1]. MVT can cause devastating intestinal hemorrhagic ischemia, peritonitis, sepsis and shock [2]. The mortality rate among patients with acute MVT ranges from 20 to 50\% [2]. A wide range of prothrombotic states have been linked to MVT, including cancer, trauma, intraabdominal inflammatory conditions, the postoperative state, oral contraceptive use, in vitro fertilization-embryo transfer (IVF-ET), cirrhosis and portal hypertension, and those caused by heritable or acquired factors, including deficiencies of protein C,

\footnotetext{
*Correspondence: doctorlipingli@foxmail.com

Department of Obstetrics and Gynecology, Guangzhou First People's

Hospital, School of Medicine, South China University of Technology,

Guangzhou, China
}

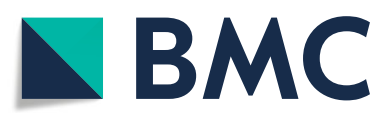

(c) The Author(s). 2018 Open Access This article is distributed under the terms of the Creative Commons Attribution 4.0 International License (http://creativecommons.org/licenses/by/4.0/), which permits unrestricted use, distribution, and

reproduction in any medium, provided you give appropriate credit to the original author(s) and the source, provide a link to the Creative Commons license, and indicate if changes were made. The Creative Commons Public Domain Dedication waiver (http://creativecommons.org/publicdomain/zero/1.0/) applies to the data made available in this article, unless otherwise stated. protein S or antithrombin III [2-4]. Antiphospholipid syndrome (APS) is a systemic autoimmune disease characterized by thrombosis-related clinical disorders or pregnancy morbidity in the presence of antiphospholipid antibodies [5]. APS has been reported to be associated with an increased risk for MVT [6, 7].

Pregnancy itself is an acquired hypercoagulable state. Occurrence of MVT in pregnancy remains reportable with few cases described in the literature. We here describe a pregnant patient who presented with an acute abdomen that was ultimately diagnosed with acute MVT after exploratory laparotomy.

\section{Case presentation}

A 26-year-old primigravid woman, at 35 weeks gestation attended our obstetric department complaining of abdominal pain along with nausea and vomiting for $3 \mathrm{~h}$. 
The woman's antenatal care was uneventful. She had no significant medical, surgical or family history and no history suggestive of thromboembolism. She had never used oral contraceptives or any other hormonal therapy.

Upon arrival, she had a temperature of $36.8^{\circ} \mathrm{C}$, pulse rate of 80 beats per minute, respiratory rate of 20 breaths per minute and blood pressure of 119/71 $\mathrm{mmHg}$. A physical examination on admission showed a gravid uterus just below the xiphoid process. No abdominal tenderness or rebound tenderness were appreciated. The bowel sounds were normal and there were no signs suggestive of peritonitis. A hematologic examination revealed a leukocyte count of $13.1 \times 10^{9} / \mathrm{L}$ (normal range $\left.4.0 \times 10^{9} / \mathrm{L}-10.0 \times 10^{9} / \mathrm{L}\right)$ with neutrophils accounting for $73.9 \%$ (normal range $50-70 \%$ ), hemoglobin level of $98.6 \mathrm{~g} / \mathrm{L}$ (normal range $100 \mathrm{~g} / \mathrm{L}-150 \mathrm{~g} / \mathrm{L}$ ), hematocrit of 0.317 (normal range 0.37-0.43), and platelet count of $187 \times 10^{9} / \mathrm{L}$ (normal range $100 \times 10^{9} / \mathrm{L}-300 \times 10^{9} / \mathrm{L}$ ). Coagulation profile and biological tests were within normal limits. Obstetric ultrasound revealed a normal fetus compatible with expected gestational age. The fetal monitor showed that fetal heart rate fluctuated between 150 and 160 beats per min and the uterus contracted occasionally. Threatened preterm labor was initially suspected and magnesium sulfate was given to inhibit uterine contractions. Acute gastritis was also considered.

The second day after admission, the patient complained of more intense abdominal pain that was centered in the right lower quadrant, and she experienced increased vomiting and abdominal distention. Physical examination demonstrated right lower quadrant tenderness without rebound tenderness and a distended abdomen and weak bowel sounds. Hematologic testing revealed leukocytosis with a left shift (leucocyte count of $26.9 \times 10^{9} / \mathrm{L}$ and neutrophils accounting for $91 \%$ ) and hemoconcentration (hematocrit of 0.439). Serum amylase was normal. An abdominal ultrasound scan revealed dilation and effusion of the right intestinal canals, thickening of the intestinal wall and a small amount of ascites. Abdominal plain radiography was performed and showed no dilated bowel loops and no features of obstruction or pneumoperitoneum. Obstetric ultrasound revealed demise of the fetus.

Acute appendicitis with perforation was suspected and an emergency exploratory laparotomy was performed immediately. On exploration, approximately $1500 \mathrm{~mL}$ of serosanguineous peritoneal fluid was found in the abdomen. The entire ileum, part of the jejunum and part of the ascending colon were gangrenous, and thromboembolism in the corresponding mesenteric veins was noted. The necrotic intestine, measuring approximately $180 \mathrm{~cm}$, was resected and an end-to-end jejunum-colon anastomosis was performed. Since there was no evidence of pending spontaneous labor and delivery and to avoid the release of fetally-derived necrotic materials that could complicate the clinical scenario via pro-coagulant and pro-inflammatory effects, a cesarean section was performed and the fetus and placenta were removed. The patient's hematological values during the operation showed a white cell count of $14.58 \times 10^{9} / \mathrm{L}$, neutrophils accounting for $91 \%$, hemoglobin of $73 \mathrm{~g} / \mathrm{L}$, a hematocrit of 0.236 and a platelet count of $135 \times 10^{9} / \mathrm{L}$. The pathologic examination revealed extensive mucosal denudation, edema, hemorrhage and neutrophil infiltration in the submucosa and muscularis propria of the bowel (Fig. 1a), and widespread thrombosis in mesenteric venous lumens (Fig. 1b).

The patient was transferred to the intensive care unit after surgery. Total parenteral nutrition, intravenous antibiotics and full anticoagulation with low molecular weight heparin were initiated postoperatively and continued until discharge. Thrombolytic therapy was withheld due to bleeding risk. The patient had a reasonably uneventful recovery and was discharged on postoperative day 36. Four years later, the patient was again pregnant and underwent thrombophilia testing (antithrombin, protein $\mathrm{C}$, protein $\mathrm{S}$, and coagulation parameters) at 30 weeks of gestation. At that time, antithrombin III activity was $64 \%$ (normal range $80-120 \%$ ). Her antithrombin III activity returned to normal (96\%) 4 weeks later. She delivered a healthy baby through a cesarean section at 38 weeks of gestation without thromboembolic event and without thromboprophylaxis. She has been recurrence-free for 10 years without anticoagulant therapy.

\section{Discussion and conclusions}

MVT is an infrequent condition accounting for 1 in 5000 to 15,000 inpatient admissions, 1 in 1000 emergency department admissions, and 6 to $9 \%$ of all cases of acute mesenteric ischemia [8]. MVT is associated with the typical components of Virchow's triad: hypercoagulability, blood stasis and vascular endothelial injury [8]. Pregnancy is an acquired hypercoagulable state that is associated with an increased risk of thromboembolism $[8,9]$. During pregnancy, factors VII, VIIIC, and fibrinogen are increased, while the activity of the fibrinolytic system is decreased [10]. In addition, as pregnancy progresses, the enlarged uterus compresses the inferior vena cava. The resulting increase in blood flow stasis adds to the pro-thrombogenic risk [11].

Still, MVT is very rare during pregnancy. We could find only 14 documented cases of MVT in pregnancy in the English literature after searching MEDLINE using keywords "mesenteric venous thrombosis" and "pregnancy" until June 30, 2018. Of these, 10 cases had precipitating factors in addition to pregnancy and 4 cases were idiopathic. As shown in Table 1, four of these 


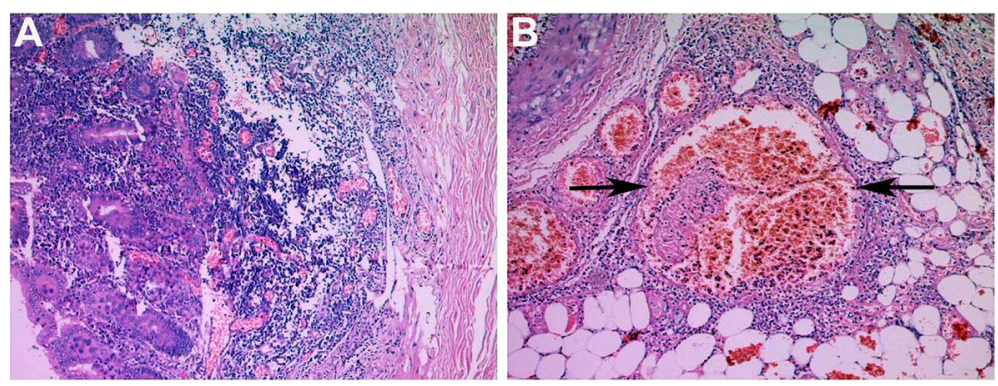

Fig. 1 Histopathological images of infarcted intestine and thrombosed mesenteric vein. (a) Presence of extensive mucosal denudation and widespread edema, hemorrhage and neutrophil infiltration in the submucosa and muscularis propria of the bowel. (b) Presence of thrombosed mesenteric vein (arrow). The sections were stained with hematoxylin and eosin (magnification: $\times 50$ )

affected pregnant women had a documented inherited thrombophilia, including factor $\mathrm{V}$ Leiden gene mutation [12], protein $\mathrm{S}$ deficiency $[13,14]$ and antithrombin deficiency [15]. One woman developed MVT following impregnation via IVF-ET [9], one had mistakenly used oral contraceptive pill and smoked heavily [16, 17], two had surgical emergencies including mid-gut volvulus [18] and a mesenteric cyst [19], one had cytomegalovirus (CMV) infection [20], one had a history of chronic idiopathic MVT [21] and the other four cases had no documented additional risk factors for MVT [10, 11, 22, 23]. Although in our reported case, antithrombin III activity was mildly decreased at a single time point during the patient's second pregnancy, it returned to normal a month later without any medication. Since the patient had no family history of inherited antithrombin III deficiency and we did not test antithrombin III activity during her first pregnancy, we believe that the occurrence of MVT during her first pregnancy was idiopathic and not secondary to deficiency of antithrombin III (Table 1).

MVT often becomes part of an ever-worsening pathogenic feedback loop. MVT impedes blood return to the

Table 1 Incidence of MVT during pregnancy

\begin{tabular}{|c|c|c|c|c|c|c|c|c|}
\hline Case & Ref. & Year & Additional risk & $\begin{array}{l}\text { Maternal } \\
\text { age }\end{array}$ & $\begin{array}{l}\text { Gestational } \\
\text { age (week) }\end{array}$ & $\begin{array}{l}\text { Intestinal } \\
\text { resection }\end{array}$ & $\begin{array}{l}\text { Anti- } \\
\text { coagulation }\end{array}$ & Pregnancy outcome \\
\hline 1 & Sönmezer, et al. [12] & 2004 & $\begin{array}{l}\text { factor } V \text { Leiden gene } \\
\text { mutation }\end{array}$ & 32 & 27 & No & Yes & $\begin{array}{l}\text { Vaginal delivery at } 39 \\
\text { weeks }\end{array}$ \\
\hline 2 & Atakan, et al. [13] & 2009 & Protein S deficiency & 25 & 20 & Yes & Yes & Maternal death \\
\hline 3 & Liu, et al. [14] & 2014 & Protein S deficiency & 38 & 7 & Yes & Yes & $\begin{array}{l}\text { Vaginal delivery of a } \\
\text { normal baby }\end{array}$ \\
\hline 4 & García-Botella, et al. [15] & 2016 & $\begin{array}{l}\text { Antithrombin } \\
\text { deficiency }\end{array}$ & 29 & 7 & Yes & Yes & $\begin{array}{l}\text { Vaginal delivery at } 36 \\
\text { weeks gestation }\end{array}$ \\
\hline 5 & Hirata, et al. [9] & 2017 & $\begin{array}{l}\text { Oral estrogen } \\
\text { associated with IVF-ET }\end{array}$ & 34 & 7 & Yes & Yes & Elective abortion \\
\hline 6 & $\begin{array}{l}\text { Friedman, et al., Graubard and } \\
\text { Friedman }[16,17]\end{array}$ & $\begin{array}{l}1984 \\
1987\end{array}$ & Oral contraceptive & 30 & 14 & Yes & Yes & ND \\
\hline 7 & Shui, et al. [18] & 2011 & Mid-gut volvulus & 25 & 35 & No & Yes & $\begin{array}{l}\text { Cesarean delivery at } 35 \\
\text { weeks gestation }\end{array}$ \\
\hline 8 & Giannos, et al. [19] & 2017 & Mesenteric cyst & 27 & 10 & Yes & Yes & Incomplete abortion \\
\hline 9 & Zamani, et al. [20] & 2009 & CMV infection & 22 & 31 & Yes & Yes & Fetal death \\
\hline 10 & Chan, et al. [21] & 2009 & Chronic idiopathic MVT & 26 & 7 & Yes & Yes & Elective abortion \\
\hline 11 & Engelhardt and Kerstein [11] & 1989 & Idiopathic & 32 & 10 & Yes & Yes & $\begin{array}{l}\text { Vaginal delivery of a } \\
\text { normal baby }\end{array}$ \\
\hline 12 & Foo, et al. [22] & 1996 & Idiopathic & 27 & 6 & No & Yes & Elective abortion \\
\hline 13 & Fouad, et al. [10] & 2001 & Idiopathic & 35 & 28 & Yes & Yes & $\begin{array}{l}\text { Vaginal delivery at } 40 \\
\text { weeks gestation }\end{array}$ \\
\hline 14 & Lin, et al. [23] & 2011 & Idiopathic & 31 & 34 & Yes & Yes & $\begin{array}{l}\text { Cesarean delivery at } 34 \\
\text { weeks gestation }\end{array}$ \\
\hline 15 & Current report & 2018 & Idiopathic & 26 & 35 & Yes & Yes & Fetal death \\
\hline
\end{tabular}

CMV, cytomegalovirus; IVF-ET, in vitro fertilization and embryo transfer; MVT, mesenteric venous thrombosis; ND, not described 
heart, leading to systemic circulatory insufficiency. Thrombosis also causes venous engorgement and bowel ischemia. Rapid and complete occlusion of the mesenteric veins can combine with the inability to expeditiously develop a compensatory collateral circulation to cause transmural bowel infarction. Transmural infarction then promotes the collection of a substantial amount of blood and fluid exudate in the intestinal lumen and peritoneal cavity, thereby fostering hemoconcentration and worsening circulatory insufficiency. Transmural infarction also induces a loss of bowel mucosal integrity, that, in turn allows for bacterial translocation. This can ultimately cause peritonitis, sepsis, multiorgan failure, septic shock, and even death [8].

The hallmark of acute MVT is abdominal pain that is typically described as colicky and midabdominal $[2,8]$. Initially, the pain may be mild. In fact, initial physical findings may be entirely normal. With the progression of the disease, the pain can become quite severe but often remains out of proportion to physical signs $[2,8]$. Nausea, anorexia, vomiting, and diarrhea are common. Some patients may experience hematemesis, hematochezia, or melena $[2,8]$. Abdominal signs such as abdominal tenderness, abdominal distension and ascites may accompany increasing bowel ischemia $[2,8]$. Fever, leukocytosis and signs of peritoneal irritation, including rebound tenderness and rigidity, develop later and indicate transmural infarction, bowel gangrene and peritonitis [2, 8]. Dehydration and hemodynamic instability characterized by hypotension, tachycardia and elevated hematocrit are very late manifestations, resulting from the collection of fluid in the bowel lumen and the abdominal cavity $[2,8]$.

The symptoms of MVT are often remarkably insidious and non-specific. Patients who present with abdominal pain may be mistakenly thought to have gastritis or peptic ulcer, and those with diarrhea may be assumed to have enteritis. When the abdominal pain becomes severe, pancreatitis is often suspected. The early symptoms of MVT in a pregnant woman are often wrongfully interpreted as normal changes of pregnancy [24] or more common pregnancy complications such as spontaneous abortion, preterm delivery, placental abruption, or uterine rupture [19]. Laboratory findings such as leukocytosis and elevated hematocrit are not particularly helpful in winnowing the differential diagnosis [4].

Abdominal plain films show non-specific findings in 50 to $70 \%$ of MVT; these include dilated bowel loops, ileus, and thumbprinting from mucosal edema [8]. Contrast enhanced computed tomography (CT) is the diagnostic modality of choice $[4,8]$. The diagnostic radiologic finding in MVT is the presence of a venous thrombus manifested as a filling defect in the mesenteric vein. The accuracy of $\mathrm{CT}$ is about $90 \%$ for the diagnosis of MVT [8]. Other findings suggesting intestinal infarction include bowel wall thickening of greater than $3 \mathrm{~mm}$, thickened mesentery, and indistinct bowel margins [8]. Ascites may also be noted [8]. Homogeneous bowel enhancement along with bowel wall thickening of $10 \mathrm{~mm}$ or more has an approximately $90 \%$ accuracy for identification of transmural infarction [8]. Bedside Doppler ultrasound can be used to detect large venous thrombi, but this diagnostic modality is operator-dependent and not as sensitive as CT [4]. Magnetic resonance (MR) imaging is another viable testing option, but it offers no distinctive advantage over CT [21]. In addition, the use of MR is limited by expense [8]. Other choices include scintiangiography and angiography, although these are limited by their low sensitivity, restricted availability and invasive nature $[4,8]$.

Early diagnosis of MVT is hindered by non-diagnostic symptoms, signs and laboratory results, and limited physician awareness of this rare disease. It is reported that only one-third of patients with acute mesenteric ischemia are correctly diagnosed before surgical exploration or death [25]. In the present case, the patient presented with typical abdominal pain, accompanied by nausea and vomiting. Her pain worsened the second day after admission and was out of proportion to the abdominal findings, which demonstrated abdominal tenderness but no abdominal distension, rebound tenderness or rigidity. The documentation of leukocytosis and elevated hematocrit offered no specific clues to the diagnosis of MVT. Preterm delivery, gastritis, and appendicitis rather than MVT were in the initial differential diagnosis. Initial radiologic testing with an abdominal plain film did not suggest bowel infarction. Even though the abdominal ultrasound scan revealed intestinal dilation, thickening of the bowel wall and a small amount of ascites, the treating physicians did not evaluate mesenteric venous flow or the possibility of MVT due to their unfamiliarity with this rare disease. A CT scan was not performed secondary to concerns about fetal risks; the estimated fetal exposure for a typical abdominal radiography is $2.5 \mathrm{mGy}$, but that of an abdominal CT scan is $30 \mathrm{mGy}$ [26]. Still, diagnostic radiography during pregnancy is not associated with any significant fetal adverse events [27] and necessary testing should be performed when indicated.

Management for MVT includes anticoagulation, surgery and supportive treatment. Anticoagulation with low molecular weight heparin should be initiated as soon as the diagnosis is made, including when diagnosis is delayed until surgery [4]. Although most researchers recommend maintaining anticoagulation therapy for at least 6 months after diagnosis in order to prevent the recurrence of the thrombosis, its benefit is still questioned in some reports [21]. If symptoms worsen or signs of peritonitis or perforation develop during initial management 
with anticoagulation alone, surgical intervention is warranted. Surgical resection of necrotic bowel and anastomosis are standard. Additional supportive care includes fluid supplementation to correct volume and electrolyte imbalances that result from fluid movement into the bowel lumen and abdominal cavity. Broad spectrum antibiotics are advocated to treat transmural infarction, septic thrombophlebitis, peritonitis, and/or sepsis secondary to intestinal bacterial translocation [4]. Bowel rest and nasogastric aspiration may be needed for patients with abdominal distension, ileus, and severe nausea or vomiting $[4,8]$.

MVT is extremely dangerous. It is characterized by an insidious onset and early non-specific clinical presentation but can progress very rapidly. Patients with MVT may deteriorate and die after a remarkably short time course due to sepsis, multiorgan failure and shock. In our case, fetal demise within $24 \mathrm{~h}$ of the patient's admission could have been secondary to intrauterine inflammation, compromised uterine blood supply and/or systemic sepsis. Early diagnosis of MVT and timely cesarean section may have allowed for a more favorable fetal outcome.

In summary, MVT in pregnancy is a rare, yet important cause of intestinal ischemia. Non-characteristic symptoms, signs and laboratory results lead to incorrect or late diagnosis of MVT and high maternal and fetal morbidity and mortality. When a pregnant patient presents with an acute abdomen, especially when the symptoms are out of proportion to the signs, MVT should be included in the differential diagnosis. Contrast enhanced $\mathrm{CT}$ is a viable choice for the diagnosis of MVT. Anticoagulation therapy should be initiated as soon as the diagnosis is made and surgical intervention should be performed when transmural infarction or peritonitis is suspected.

\section{Abbreviations}

APS: Antiphospholipid syndrome; CMV: cytomegalovirus; CT: computed tomography; IVF-ET: in vitro fertilization-embryo transfer; MR: Magnetic resonance; MVT: Mesenteric venous thrombosis; ND: not described

\section{Funding}

Funding was not used to complete this case report.

\section{Availability of data and materials}

The datasets used and/or analyzed during the current study are available from the corresponding author on reasonable request.

\section{Authors' contributions}

XG and LH drafted the manuscript. XG wrote the paper, and the Manuscript was reviewed and edited by LL. All authors read and approved the final manuscript.

\section{Ethics approval and consent to participate}

This case report was conducted with the approval of the Medical Ethics Committee of Guangzhou First People's Hospital and with patient consent.

\section{Consent for publication}

Written informed consent was obtained from the patient for publication of this case report and any accompanying images.

\section{Competing interests}

The authors declare that they have no competing interests.

\section{Publisher's Note}

Springer Nature remains neutral with regard to jurisdictional claims in published maps and institutional affiliations.

Received: 25 July 2018 Accepted: 28 November 2018

Published online: 11 December 2018

References

1. Acosta S, Alhadad A, Svensson P, Ekberg O. Epidemiology, risk and prognostic factors in mesenteric venous thrombosis. Br I Surg. 2008:95:1245-51.

2. Kumar S, Sarr MG, Kamath PS. Mesenteric venous thrombosis. N Engl J Med. 2001;345:1683-8.

3. Dorais J, Jones K, Hammoud A, Gibson M, Johnstone E, Peterson CM. A superior mesenteric vein thrombosis associated with in vitro fertilization. Fertil Steril 2011:95:804.e11-3.

4. Hmoud B, Singal AK, Kamath PS. Mesenteric venous thrombosis. J Clin Exp Hepatol. 2014:4:257-63.

5. Garcia D, Erkan D. Diagnosis and Management of the Antiphospholipid Syndrome. N Engl J Med. 2018;378:2010-21.

6. Fried M, Van Ganse W, Van Avermaet S. Mesenteric vein thrombosis triggered by blunt abdominal trauma in a patient with the primary antiphospholipid syndrome. Eur J Gastroenterol Hepatol. 2002;14:697-700.

7. Vacca A, Garau P, Cauli A, Mameli A, Mathieu A. Primary antiphospholipid syndrome with mesenteric venous thrombosis presenting with intestinal infarction: a case description. Lupus. 2007;16(6):455-6.

8. Singal AK, Kamath PS, Tefferi A. Mesenteric venous thrombosis. Mayo Clin Proc. 2013;88:285-94.

9. Hirata M, Yano H, Taji T, Shirakata Y. Mesenteric vein thrombosis following impregnation via in vitro fertilization-embryo transfer. World J Gastrointest Surg. 2017;9:209-13.

10. Fouad MA, Pathania AG, Marouf R. Primary mesenteric venous thrombosis in a 28-week pregnant woman. Med Principles Pract. 2001;10:204-6.

11. Engelhardt TC, Kerstein MD. Pregnancy and mesenteric venous thrombosis. South Med J. 1989;82:1441-3.

12. Sönmezer $M$, Aytaç $R$, Demirel LC, Kurtay G. Mesenteric vein thrombosis in a pregnant patient heterozygous for the factor $V(1691 \mathrm{G} \rightarrow$ a) Leiden mutation. Eur J Obstet Gynecol Reprod Biol. 2004;114:234-5.

13. Atakan Al R, Borekci B, Ozturk G, Akcay MN, Kadanali S. Acute mesenteric venous thrombosis due to protein $S$ deficiency in a pregnant woman. J Obstet Gynaecol Res. 2009;35:804-7.

14. Liu J, Tan WC, Tan EL. Yu SL. Successful management of acute mesenteric ischaemia in a pregnant woman with protein S deficiency. Ann Acad Med Singap. 2014;43:125-6.

15. García-Botella A, Asenjo S. De la Morena-barrio ME, corral J, Bolaños E, Carlin $P S$, et al. first case with antithrombin deficiency, mesenteric vein thrombosis and pregnancy: multidisciplinary diagnosis and successful management. Thromb Res. 2016;144:72-5.

16. Friedman M, Zimmer EZ, Graubard ZG. Mesenteric vein thrombosis associated with oral contraceptive administration during pregnancy. Ann Chir Gynaecol. 1984:73:296-8.

17. Graubard ZG, Friedman M. Mesenteric venous thrombosis associated with pregnancy and oral contraception. A case report S Afr Med J. 1987;71:453.

18. Shui LH, Rafi J, Corder A, Mowbray D. Mid-gut volvulus and mesenteric vessel thrombosis in pregnancy: case report and literature review. Arch Gynecol Obstet. 2011;283(Suppl 1):39-43.

19. Giannos A, Stavrou S, Goumalatsos N, Fragkoulidis G, Chra E, Argiropoulos $D$, et al. Mesenteric cysts and mesenteric venous thrombosis leading to intestinal necrosis in pregnancy managed with laparotomy: a case report and review of the literature. J Med Case Rep. 2017;11:184.

20. Zamani F, Amiri A, Mohit M, Zolfaghari M, Jalaeefar A, Ranjbar M, et al. CMV infection in a pregnant woman complicated by toxic megacolon and mesenteric vein thrombosis. Turk J Gastroenterol. 2009;20:234-5. 
21. Chan CM, Chen WL, Chen JH, Wu YL, Huang CC. Pregnancy-induced acute intestinal infarction in a woman with chronic idiopathic mesenteric vein thrombosis under regular anticoagulation treatment. Med Princ Pract. 2009:18:422-4.

22. Foo E, Sim R, Ng BK. Case report of acute splenic and superior mesenteric vein thrombosis and its successful medical management. Ann Acad Med Singap. 1996;25:755-8.

23. Lin H, Lin CC, Huang WT. Idiopathic superior mesenteric vein thrombosis resulting in small bowel ischemia in a pregnant woman. Case Rep Obstet Gynecol. 2011;2011:687250.

24. Duran R, Denys AL, Letovanec I, Meuli RA, Schmidt S. Multidetector CT features of mesenteric vein thrombosis. Radiographics. 2012;32:1503-22.

25. Mamode N, Pickford I, Leiberman P. Failure to improve outcome in acute mesenteric ischaemia: seven-year review. Eur J Surg. 1999;165:203-8.

26. Ratnapalan S, Bona N, Chandra K, Koren G. Physicians' perceptions of teratogenic risk associated with radiography and $\mathrm{CT}$ during early pregnancy. Am J Roentgenol. 2004;182:1107-9.

27. Lowe SA. Diagnostic radiography in pregnancy: risks and reality. Aust N Z J Obstet Gynaecol. 2004;44:191-6.

Ready to submit your research? Choose BMC and benefit from:

- fast, convenient online submission

- thorough peer review by experienced researchers in your field

- rapid publication on acceptance

- support for research data, including large and complex data types

- gold Open Access which fosters wider collaboration and increased citations

- maximum visibility for your research: over $100 \mathrm{M}$ website views per year

At BMC, research is always in progress.

Learn more biomedcentral.com/submissions 